Proceedings of the Edinburgh Mathematical Society (2002) 45, 421-448 (C)

DOI:10.1017/S0013091500001164 Printed in the United Kingdom

\title{
THE EULER CLASS OF A POINCARÉ DUALITY GROUP
}

\author{
IAN J. LEARY \\ Faculty of Mathematical Studies, University of Southampton, \\ Southampton SO17 1BJ, UK (I.J.Leary@maths.soton.ac.uk)
}

(Received 20 November 2000)

\begin{abstract}
Under an extra hypothesis satisfied in every known case, we show that the Euler class of an orientable odd-dimensional Poincaré duality group over any ring has order at most two. We construct groups that are of type FL over the complex numbers but are not FL over the rationals. We construct group algebras over fields for which $K_{0}$ contains torsion, and construct non-free stably free modules for the group algebras of certain virtually free groups.
\end{abstract}

Keywords: group cohomology; Poincaré duality; finiteness conditions

AMS 2000 Mathematics subject classification: Primary 19A31

Secondary 16S34; 20J05; 55N15; 57P10

\section{Introduction}

Let $G$ be a group and $R$ a (non-trivial, commutative, unital) ring. A number of finiteness conditions for the pair $R$ and $G$ have played a role in homological group theory and in algebraic topology. Classical examples include the conditions FP and FL. Both FP and FL are defined purely algebraically, although in the case when $R=\mathbb{Z}$ and $G$ is finitely presented both have topological interpretations, discovered by Wall [33,34]. Recently, Bestvina and Brady introduced a topological condition, FH, at least as strong as FL, in the course of their work exhibiting non-finitely presented groups of type FL over $\mathbb{Z}[\mathbf{5}]$. (In fact, they show that their groups are FL by showing that they are FH.)

Roughly speaking, the difference between FL and FP is measured by an element $E(G)$ in $K_{0}(R G)$, which we shall call the Euler class of $G$. More precisely, if $G$ is $\mathrm{FP}$, then the image of $E(G)$ in the reduced $K$-group $\tilde{K}_{0}(R G)$ is zero if and only if $G$ is FL. This image will be denoted $\tilde{E}(G)$ and referred to as Wall's finiteness obstruction. As general references we recommend $[\mathbf{2 4 , 3 0}]$ for $K_{0}$ and Wall's finiteness obstruction, and [8] for finiteness conditions for groups.

We introduce a new finiteness condition FPP, defined algebraically, and lying between FP and FL. (The extra ' $P$ ' stands for 'permutation'.) Just as every known torsion-free group of type FP is of type FL, it seems to be the case that every group of type FP is of type FPP. We show that the Euler class of an odd-dimensional orientable Poincaré duality group of type FPP has order at most two. In cases when $K_{0}$ has no 2-torsion, 
it follows that such a group is necessarily of type FL. We exhibit groups $G$ such that $K_{0}(\mathbb{Q} G)$ contains torsion which is mapped to zero in $K_{0}(\mathbb{C} G)$. For some of these groups, we show that $E(G)$ is a non-zero element of this kernel, and hence exhibit groups of type FP over $\mathbb{Q}$ that are FL over $\mathbb{C}$ but not FL over $\mathbb{Q}$. We deduce that over $\mathbb{C}, \mathrm{FH}$ is strictly stronger than FL.

Some of the techniques used for computing $K_{0}$ also apply to the abelian monoid $\mathcal{P}$ of isomorphism types of finitely generated projective modules. Hence we are able to give examples of non-free stably free projective modules for certain group algebras.

Most of our calculations of $K$-groups (and the monoid $\mathcal{P}$ ) rely on work of Waldhausen, or of Bergman and Dicks $[\mathbf{2}, \mathbf{1 2}, \mathbf{3 2}]$, but we do give a self-contained and comparatively elementary proof that there are groups that are FL over $\mathbb{C}$ but not $F L$ over $\mathbb{Q}$. The definitions of all of the finiteness conditions mentioned above, and statements of our main results, are contained in the next section.

\section{Definitions and statements}

For $G$ and $R$ as above, recall that $G$ is said to be of type FP over $R$ if there is a finite $R G$-projective resolution of $R$ (viewed as an $R G$-module by letting each $g \in G$ act as the identity) in which each term is finitely generated, i.e. an exact sequence

$$
0 \rightarrow P_{m} \rightarrow P_{m-1} \rightarrow \cdots \rightarrow P_{1} \rightarrow P_{0} \rightarrow R \rightarrow 0
$$

in which each $P_{i}$ is a finitely generated projective module [8, VIII.6]. Similarly, $G$ is said to be of type FL over $R$ if there is an exact sequence as in (2.1) in which each $P_{i}$ is a finitely generated free module.

We define $G$ to be of type FPP over $R$ if there exists a sequence as above in which each $P_{i}$ is a finitely generated projective permutation module, i.e. a finite direct sum of modules of the form $R G / H$, where $H$ is a finite subgroup of $G$ whose order is a unit in $R$. Clearly, for any $G$ and $R, \mathrm{FL} \Rightarrow \mathrm{FPP} \Rightarrow \mathrm{FP}$, and if $G$ is $R$-torsion-free, then FL $\Leftrightarrow \mathrm{FPP}$. The author is not aware of any $G$ and $R$ such that FP $\nRightarrow$ FPP. The existence of such $G$ and $R$ is a generalization of the question of whether there exists torsion-free $G$ and $R$ such that FP $\nRightarrow F$ F. Examples of groups of type FP include any group $G$ acting on an $R$-acyclic simplicial complex $X$ with finitely many orbits of simplices, in such a way that the stabilizer of any simplex has order a unit in $R$. Every such $G$ is FPP over $R$.

For any $G$ of type FP over $R$, the Euler class $E(G)$ is the element of $K_{0}(R G)$ represented by the alternating sum

$$
E(G)=\sum_{i=0}^{m}(-1)^{i}\left[P_{i}\right] \in K_{0}(R G),
$$

where $P_{i}$ is the $i$ th module occurring in the projective resolution (2.1), and $\left[P_{i}\right]$ stands for the corresponding class in $K_{0}(R G)$. The Euler class depends only on $(G, R)$ and not on the choice of resolution. It may be shown that $E(G)$ is in the subgroup of $K_{0}(R G)$ generated by $[R G]$, the class of the free module, if and only if $G$ is FL over $R$. Similarly, 
$G$ is FPP over $R$ if and only if $E(G)$ is in the subgroup of $K_{0}(R G)$ generated by elements of the form $[R G / H]$.

$G$ is said to be a Poincaré duality group of dimension $n$ over $R$ (or a $\mathrm{PD}^{n}$ group over $R$ ) if $G$ is $\mathrm{FP}$ over $R$ and as $R$-modules,

$$
H^{i}(G, R G) \cong \begin{cases}R, & \text { for } i=n, \\ 0, & \text { for } i \neq n\end{cases}
$$

For any $G$, the $R G$-bimodule structure on $R G$ gives rise to a right $R G$-module structure on $H^{*}(G, R G)$. A $\mathrm{PD}^{n}$ group $G$ over $R$ is said to be orientable if each element of $G$ acts as the identity on $H^{n}(G, R G)$. These definitions are equivalent to those of Bieri [6] and of Johnson and Wall [17], except that Johnson and Wall only consider finitely presented groups. Davis has recently given examples of $\mathrm{PD}^{n}$ groups over the integers, for each $n \geqslant 4$, that are not finitely presented [11]. If a group $G$ acts on an $R$-acyclic simplicial complex $X$ in the manner described above, and, moreover, $X$ is an $R$-homology $n$-manifold, then $G$ is $\mathrm{PD}^{n}$ over $R$. (A simplicial complex is an $R$-homology $n$-manifold if the link of every $i$-simplex has the same $R$-homology as an $(n-i-1)$-sphere.)

Our first main result is the following theorem.

Theorem 2.1. Let $G$ be an orientable $\mathrm{PD}^{n}$ group over $R$ for some odd $n$, and suppose that $G$ is of type FPP over $R$. Then $E(G) \in K_{0}(R G)$ has order at most two.

This theorem should be compared with the familiar fact that the Euler characteristic of a closed odd-dimensional manifold is zero. There are examples satisfying the hypotheses of the theorem for which $E(G)$ is known to have order two. The first such example was a crystallographic group, discovered by Kropholler and Moselle, with the property that $E(G)$ is an element of order two in $K_{0}(k G)$ for any field of characteristic zero [18]. The proof of Theorem 2.1 uses a generalization of an argument presented in [18].

Theorem 2.1 leads naturally to the study of torsion in $K_{0}$ of group algebras. One class of groups for which $K_{0}$ can be explicitly computed is the class of virtually free groups, to which work of Bergman, Dicks and Waldhausen applies $[\mathbf{2}, \mathbf{1 2}, \mathbf{3 1}, \mathbf{3 2}]$. Using these results we are able to give examples as in the following statement, which will be proved in $\S 6$.

Theorem 2.2. For every integer $n$, and every field $k$ of characteristic zero, there is a virtually free group $G$ for which $K_{0}(k G)$ contains an element of order $n$. For every prime $p$, there is a virtually free group $G$ for which $K_{0}(\mathbb{Q} G)$ contains an element of order $p$, which is mapped to zero in $K_{0}(\mathbb{C} G)$.

The only published examples of torsion in $K_{0}(k G)$ seem to be the examples due to Kropholler and Moselle [18], those contained in a recent paper of Lorenz [22], and our example in $[\mathbf{1 9}]$. In $\left[\mathbf{1 8 , 2 2}\right.$, crystallographic groups are given for which $K_{0}(k G)$ contains $2-, 3$ - and 4 -torsion, for any field $k$ of characteristic zero. In [19], a virtually cyclic group $G$ was exhibited, for which there is an element of order two in $K_{0}(\mathbb{Q} G)$ mapping to zero in $K_{0}(\mathbb{C} G)$. 
Of course, virtually free groups are rarely Poincaré duality groups, but virtually free quotients of a Poincaré duality group $G$ can be used to detect elements of $K_{0}(k G)$. Moreover, a relative version of Theorem 2.1 can be given (see Theorem 8.2 for an exact statement), which is applicable to some virtually free groups. Using these ideas we prove the following statement, in $\S \S 5,7$ and 8 .

Theorem 2.3. The following classes contain examples of groups that are FL over the complex numbers but not FL over the rationals:

(a) orientable crystallographic groups of dimension three;

(b) orientable $\mathrm{PD}^{3}$ groups over the rationals containing non-cyclic free subgroups; and

(c) virtually free groups.

A 'suspension lemma', stated and proved as Corollary 7.3, allows one to deduce the following corollary.

Corollary 2.4. For each $n \geqslant 3$ there are $\mathrm{PD}^{n}$ groups over the rationals that are FL over the complex numbers but not FL over the rationals.

In both Theorems 2.2 and 2.3, more information concerning the behaviour under field extensions can be given, and will be given below. The simplest of the examples in Theorem 2.3 is the index-two orientable subgroup of the group of reflections in the sides of a right regular triangular prism in $\mathbb{R}^{3}$. For this $G$, and any field $k$ of characteristic not equal to 2 or 3 , we compute $K_{0}(k G)$ in Theorem 5.2. It transpires that $G$ is of type FL if and only if $k$ contains a primitive cube root of 1 . The calculation for this example is modelled on the one in [18], but is complicated by the fact that we need to use Moody's Theorem $[\mathbf{2 5}]$ (as well as $[\mathbf{2}, \mathbf{1 2}, \mathbf{3 1}]$ ).

In the proof of parts (a) and (b) of Theorem 2.3, the dependence on the work of Bergman, Dicks and Waldhausen can be reduced to showing that a certain projective module for a certain algebra (a quotient of each of the relevant group algebras) is not stably free. In [19] we gave an elementary proof of this fact using well-known facts about topological $K$-theory. In $\S 10$ we give a similar but more algebraic proof of this, by reducing to a computation in $K_{0}$ of a Dedekind domain.

Groups having similar properties to the examples given in Theorems 2.2 and 2.3 were obtained by Moselle using different methods. Moselle's thesis contained a detailed study of $K_{0}$ of the rational group algebras of groups of the form $I G: G$, where $G$ is finite and $I G$ is the augmentation ideal in $\mathbb{Z} G$, viewed as a $G$-module [26]. Using a theorem of Quinn, given in $[\mathbf{2 9}]$, Moselle exhibited groups of type FL over $\mathbb{C}$ but not of type FL over $\mathbb{Q}$, and groups for which $E(G) \in K_{0}(\mathbb{Q} G)$ has order $p$ for any prime $p[\mathbf{2 6}]$. No full proof of Quinn's theorem has appeared, and we consider it an advantage that our results do not depend upon it.

Bestvina and Brady say that $G$ is of type FH over $R$ if there is an $R$-acyclic simplicial complex upon which $G$ acts freely with finitely many orbits [5]. For any $G$ and $R$, $\mathrm{FH} \Rightarrow \mathrm{FL}$. In $\S 9$ we point out that $\mathrm{FH}$ is insensitive to field extensions, and hence that the groups listed in Theorem 2.3 cannot be of type FH over the complex numbers. 
In contrast, we show that over the rationals, $\mathrm{FL} \Leftrightarrow \mathrm{FH}$ for groups having finite-type Eilenberg-Mac Lane spaces.

The techniques that we use to compute $K_{0}$ for virtually free groups also allow one to describe the abelian monoid of finitely generated projective modules. In $\S 11$ we use these techniques to exhibit virtually free groups $G$ for which there are non-free stably free $k G$-modules for any field $k$ of characteristic zero.

\section{Duality and Euler classes}

If $M$ is an $R G$-module, the $R G$-bimodule structure on $R G$ gives rise to a right $R G$ module structure on $\operatorname{Hom}_{R G}(M, R G)$. Define $M^{*}$ to be the $R G$-module obtained from $N=\operatorname{Hom}_{R G}(M, R G)$ by defining $g n=n g^{-1}$ for any $g \in G$ and $n \in N$. The assignment $M \mapsto M^{*}$ is a contravariant functor. If $M$ is a finitely generated projective module, then $M^{* *}$ is naturally isomorphic to $M$.

Proposition 3.1. Let $H$ be a finite subgroup of $G$. Then $(R G / H)^{*} \cong R G / H$.

Proof. It suffices to show that $\operatorname{Hom}_{R G}(R G / H, R G) \cong R H \backslash G$, the right permutation module on the right cosets of $H$. But an element $f$ in $\operatorname{Hom}_{R G}(R G / H, R G)$ is determined uniquely by $f(1 . H)=\sum_{g} r_{g} g$, and this can be any element of $R G$ fixed by the action of $H$, i.e. any element such that $r_{g}=r_{h g}$ for all $h \in H$ and $g \in G$. Let $T$ be a right transversal to $H$ in $G$. The set of elements of this form is right $R G$-isomorphic to $R H \backslash G$ via the map

$$
\sum_{g \in G} r_{g} g \mapsto \sum_{t \in T} r_{t} H t, \quad \text { with inverse } \sum_{t \in T} r_{t} H t \mapsto \sum_{t \in T} \sum_{g \in H t} r_{t} g .
$$

Lemma 3.2. Let $G$ be a group of type FPP over $R$. Then there is a resolution of $R$ over $R G$ by projective permutation modules of length equal to the cohomological dimension of $G$ over $R$.

Proof. Let $n$ denote the cohomological dimension of $G$ over $R$. Since $G$ is FPP over $R$, there is a resolution

$$
0 \rightarrow P_{m} \rightarrow P_{m-1} \rightarrow \cdots \rightarrow P_{1} \rightarrow P_{0} \rightarrow R \rightarrow 0
$$

for the trivial $R G$-module in which each $P_{i}$ is a finitely generated projective permutation module. Necessarily, $m \geqslant n$, but equality need not hold. However, since $\{0\}$ is a projective permutation module, we may assume that $m-n$ is even, which will simplify the notation in the remainder of the proof.

The image of the map from $P_{n}$ to $P_{n-1}$ is a finitely generated projective, $Q_{n}$, and there are long exact sequences

$$
\begin{aligned}
& 0 \rightarrow P_{m} \rightarrow P_{m-1} \rightarrow \cdots \rightarrow P_{n} \rightarrow Q_{n} \rightarrow 0, \\
& 0 \rightarrow Q_{n} \rightarrow P_{n-1} \rightarrow \cdots \rightarrow P_{1} \rightarrow P_{0} \rightarrow R \rightarrow 0 .
\end{aligned}
$$


It will suffice to show that $Q_{n}$ is stably a projective permutation module in the sense that there is a finitely generated projective permutation module $P^{\prime}$ such that $P^{\prime} \oplus Q_{n}$ is also a projective permutation module, since then the direct sum of sequence (3.2) and an exact sequence consisting of two copies of $P^{\prime}$, in degrees $n-1$ and $n$, will be a resolution with the required properties.

Each term in sequence (3.1) is projective, so the sequence splits, and hence there is an isomorphism

$$
Q_{n} \oplus \bigoplus_{i=1}^{(m-n) / 2} P_{n-1+2 i} \cong \bigoplus_{i=1}^{(m-n) / 2} P_{n+2 i} .
$$

The module $P^{\prime}=\bigoplus_{i=1}^{(m-n) / 2} P_{n-1+2 i}$ therefore has the properties that both $P^{\prime}$ and $P^{\prime} \oplus$ $Q_{n}$ are finitely generated projective permutation modules as required.

Proof of Theorem 2.1. By Lemma 3.2 we may take a length $n$ resolution of $R$ by finitely generated projective permutation modules:

$$
0 \rightarrow P_{n} \rightarrow P_{n-1} \rightarrow \cdots \rightarrow P_{1} \rightarrow P_{0} \rightarrow R \rightarrow 0 .
$$

Since $G$ is an orientable Poincaré duality group of dimension $n$ over $R$, applying $(-)^{*}$ to this resolution gives rise to an exact sequence

$$
0 \rightarrow P_{0}^{*} \rightarrow P_{1}^{*} \rightarrow \cdots \rightarrow P_{n-1}^{*} \rightarrow P_{n}^{*} \rightarrow R \rightarrow 0 .
$$

By Proposition 3.1, $P_{i} \cong P_{i}^{*}$ for each $i$. Sequence (3.3) gives the expression

$$
E(G)=\sum_{i=0}^{n}(-1)^{i}\left[P_{i}\right]
$$

for $E(G)$. On the other hand, sequence (3.4) gives

$$
E(G)=\sum_{j=0}^{n}(-1)^{j}\left[P_{n-j}^{*}\right]=\sum_{i=0}^{n}(-1)^{n-i}\left[P_{i}\right] .
$$

Since $n$ is odd, it follows that $E(G)=-E(G)$, as required.

Theorem 2.1, and its proof, are closely related to Theorem 1.3 of Wall's paper [35]. Wall mentions only the case $R=\mathbb{Z}$, but the generalization to any $R$ is immediate. On the other hand, Wall does not impose any condition corresponding to our FPP, so obtains a weaker conclusion.

Under certain circumstances Theorem 2.1 may be used to show that a Poincaré duality group is of type FL.

Corollary 3.3. Let $G$ contain only finitely many conjugacy classes of finite subgroups, let $k$ be a field which is a splitting field for every finite subgroup of $G$, and suppose that $G$ contains no element of order two. Suppose also that either (a) $G$ is polycyclic-by-finite or (b) $k$ has characteristic zero. If $G$ is an orientable $\mathrm{PD}^{n}$ group over $k$ of type FPP for some odd n, then $G$ is FL. 
Proof. First, note that the condition that $G$ be of type FPP over $k$ implies that the order of every finite subgroup of $G$ is invertible in $k$. For any $G$, let $\Phi(G)$ be the Frobenius category of $G$. The objects of $\Phi$ are the finite subgroups of $G$, and the morphisms from $H$ to $H^{\prime}$ are those group homomorphisms from $H$ to $H^{\prime}$ that are expressible as conjugation by some element of $G$. For any $k$, the natural maps $K_{0}(k H) \rightarrow K_{0}(k G)$ induce a map

$$
\iota: \operatorname{colim}_{H \in \Phi} K_{0}(k H) \rightarrow K_{0}(k G) .
$$

If $G$ is of type FPP over $k$, the Euler class of $G$ is in the image of $\iota$.

Now suppose that $G$ and $k$ satisfy the hypotheses of the first sentence of the statement. In this case, work of Brown and Lorenz shows that $C=\operatorname{colim}_{H \in \Phi} K_{0}(k H)$ is a finitely generated abelian group containing no 2-torsion $[\mathbf{9}, 2.5]$. The rank of $C$ (i.e. the dimension of the vector space $C \otimes \mathbb{Q}$ ) is equal to the number of conjugacy classes of elements of finite order in $G$ (see $[\mathbf{9}, \S 1]$ or $[\mathbf{2 3}, \S 8])$.

Two different arguments may be used to show that the rank of the image of $\iota$ is equal to the rank of $C$, depending on whether condition (a) or (b) is satisfied. In the case when $k$ is of characteristic zero, Hattori-Stallings ranks may be used to show that the rank of the image of $\iota$ is equal to the number of conjugacy classes of elements of finite order in $G$. In the case when $G$ is polycyclic-by-finite, Lorenz has shown that $G$ admits a finite quotient $Q$ such that the induced map of Frobenius categories $\Phi(G) \rightarrow \Phi(Q)$ is full (i.e. is surjective on morphism sets) (see Lemma 1 of $[\mathbf{2 1}]$ and $\S 1.6$ of [9]. In this case it may be shown that the kernel of map from $C$ to $K_{0}(k Q)$ (which factors through $\iota)$ is finite $[\mathbf{9 , 2 1}]$. In each of cases (a) and (b), the kernel of $\iota$ is finite, and it follows that there is no 2-torsion in the image of $\iota$.

Now suppose that $G$ and $k$ also satisfy the hypotheses of the final sentence of the statement. By Theorem 2.1, $E(G)$ has order at most two, but by the above argument $E(G)$ cannot have order two, and so $G$ is FL.

The author is not aware of any group $G$ and field $k$ for which the natural map $\iota$ : $\operatorname{colim}_{H \in \Phi(G)} K_{0}(k H) \rightarrow K_{0}(k G)$ described in the above proof is not an isomorphism.

\section{Computational techniques}

In this section we summarize the techniques for computing $K_{0}(R G)$ that will be used in the sequel. For the definition of a graph of groups, see [13]. The following theorem is from $[\mathbf{3 1}]$, but see also $[\mathbf{2}, \mathbf{1 2}, \mathbf{3 2}]$.

Theorem 4.1 (Waldhausen). Let $G$ be the fundamental group of a finite graph of groups, with vertex groups $G_{1}, \ldots, G_{m}$ and edge groups $H_{1}, \ldots, H_{n}$. Let $k$ be a field and suppose that each $k H_{j}$ is regular Noetherian. There is an exact sequence

$$
\bigoplus_{j=1}^{n} K_{0}\left(k H_{j}\right) \stackrel{\iota}{\rightarrow} \bigoplus_{i=1}^{m} K_{0}\left(k G_{i}\right) \rightarrow K_{0}(k G) \rightarrow 0,
$$

where the map $\iota$ is induced by the inclusion of each edge group in its initial vertex group minus the inclusion in its terminal vertex group. 
Remarks on the proof. The conditions on the $k H_{j}$ hold if each $H_{j}$ is polycyclicby-finite and the order of every finite subgroup of every $H_{j}$ is invertible in $k$. (This is the only case in which we shall use the theorem.) The theorem may be proved by induction on the size of the graph, using $\S \S 11$ and 12 of [32], which cover the cases of a free product with amalgamation and an HNN (Higman-Neumann-Neumann)-extension, respectively. The result required for exactness at $K_{0}(k G)$ is not explicitly stated in $\S 12$, but works in the same way as similar results stated in $\S \S 11$ and 13 of [32].

In the case when each $H_{i}$ is finite, which will serve for all of our calculations except those in $\S 5$, Theorem 4.1 may be deduced from work of Bergman and Dicks, see in particular Corollary 2.11 of $[\mathbf{2}]$ and Theorem 26 of $[\mathbf{1 2}]$, which cover the case of a free product with amalgamation and an HNN-extension, respectively. The alternative approach of Bergman-Dicks also describes the abelian monoid of finitely generated projective $k G$ modules. This will be used in $\S 11$ to exhibit non-free stably free modules. A shorter statement of Theorem 4.1 is the following. Make a category $\mathcal{C}$ whose objects are the vertex and edge groups, with two non-identity morphisms from each edge group, the homomorphisms to its initial and terminal vertex groups. Then the following map is an isomorphism:

$$
\operatorname{colim}_{H \in \mathcal{C}} K_{0}(k H) \rightarrow K_{0}(k G) .
$$

The graphs of groups used below are of a particularly simple type. These graphs are described by a group $J$ and a family $H_{1}, \ldots, H_{n}$ of (not necessarily distinct) subgroups of $J$. There are two vertex groups, $J_{1}$ and $J_{2}$, identified with $J$ by some fixed isomorphism, and edge groups $H_{1}, \ldots, H_{n}$. Each edge joins the two vertices, and the map from $H_{i}$ to $J_{j}$ is the inclusion of $H_{i}$ in $J$. Call the fundamental group of such a graph the double of $J$ along $H_{1}, \ldots, H_{n}$.

Proposition 4.2. Let $G$ be the double of a polycyclic-by-finite group $J$ along the subgroups $H_{1}, \ldots, H_{n}$. Let $k$ be a field in which the order of every finite subgroup of $J$ is invertible. Then there are isomorphisms

$$
K_{0}(k G) \cong K_{0}(k J) \oplus \operatorname{coker}\left(\iota: \bigoplus_{i=1}^{n} K_{0}\left(k H_{i}\right) \rightarrow K_{0}(k J)\right)
$$

and

$$
\tilde{K}_{0}(k G) \cong \tilde{K}_{0}(k J) \oplus \operatorname{coker}\left(\iota: \bigoplus_{i=1}^{n} K_{0}\left(k H_{i}\right) \rightarrow K_{0}(k J)\right) .
$$

Under these hypotheses, $G$ is of type FP over $k$, and the above isomorphism sends $E(G) \in K_{0}(k G)$ to the element

$$
\left(2 E(J)-\sum_{i=1}^{n} \operatorname{Ind}_{H_{i}}^{J} E\left(H_{i}\right), E(J)\right) \in K_{0}(k J) \oplus \operatorname{coker}(\iota) .
$$

Proof. Let $A=K_{0}(k J)$. By Theorem 4.1, $K_{0}(k G)$ is isomorphic to the quotient $(A \oplus A) /\{(\iota(x),-\iota(x))\}$, where $x \in \bigoplus K_{0}\left(k H_{i}\right)$. The automorphism of $A \oplus A$ given by 
$\left(a, a^{\prime}\right) \mapsto\left(a+a^{\prime}, a^{\prime}\right)$ induces an isomorphism between this and $(A \oplus A) /\{(0,-\iota(x))\}=$ $A \oplus \operatorname{coker}(\iota)$.

The augmented chain complex for the $G$-tree coming from the graph product decomposition is the following exact sequence:

$$
0 \rightarrow \bigoplus_{i=1}^{n} k G / H_{i} \rightarrow k G / J_{1} \oplus k G / J_{2} \rightarrow k \rightarrow 0 .
$$

Our hypothesis on the orders of the finite subgroups of $J$ implies that $J$ and all the $H_{i}$ are of type FP over $k$ (see $[\mathbf{2 8}, 10.3 .13]$ ). The above exact sequence implies that $G$ is also of type FP over $k$. Moreover, it follows that the Euler class of $G$ is the sum of the images of $E\left(J_{1}\right)$ and $E\left(J_{2}\right)$ minus the sum of the images of the $E\left(H_{i}\right)$. Tracing this element through the isomorphism given in the first paragraph gives the claimed element of $A \oplus \operatorname{coker}(\iota)$.

It is possible to prove some results concerning doubles without using Theorem 4.1. In the following proposition we need no condition on $J$ except that it be FP over $R$.

Proposition 4.3. Let $H \leqslant J$ be groups of type FP over $R$. Let $G$ be the double of $J$ along $H$, so that $G$ is $\mathrm{FP}$ over $R$. If $E(J) \in K_{0}(R J)$ is in the image of $\operatorname{Ind}_{H}^{J}$ and $\operatorname{Ind}_{H}^{J}(E(H))=2 E(J)$, then $E(G)=0$.

Proof. As in Proposition 4.2, consideration of the chain complex for the $G$-tree coming from the given splitting shows that

$$
E(G)=\operatorname{Ind}_{J_{1}}^{G}\left(E\left(J_{1}\right)\right)+\operatorname{Ind}_{J_{2}}^{G}\left(E\left(J_{2}\right)\right)-\operatorname{Ind}_{H}^{G}(E(H)),
$$

where $J_{1}$ and $J_{2}$ are the two copies of $J$ in $G$. If $E(J)=\operatorname{Ind}_{H}^{J}(\xi)$, then

$$
\begin{aligned}
E(G) & =\operatorname{Ind}_{J_{1}}^{G}\left(E\left(J_{1}\right)\right)+\operatorname{Ind}_{J_{2}}^{G} \operatorname{Ind}_{H}^{J_{2}}(\xi)-\operatorname{Ind}_{H}^{G}(E(H)) \\
& =\operatorname{Ind}_{J_{1}}^{G}\left(E\left(J_{1}\right)\right)+\operatorname{Ind}_{H}^{G}(\xi-E(H)) \\
& =\operatorname{Ind}_{J_{1}}^{G}\left(E\left(J_{1}\right)+\operatorname{Ind}_{H}^{J_{1}}(\xi-E(H))\right) \\
& =\operatorname{Ind}_{J_{1}}^{G}\left(2 E\left(J_{1}\right)-\operatorname{Ind}_{H}^{J_{1}}(E(H))\right) .
\end{aligned}
$$

There is an argument similar to that used in the proof of Theorem 2.1 to exhibit some cases in which $\operatorname{Ind}_{H}^{J}(E(H))=2 E(J)$. For a Poincaré duality group $G$ over $R$, the orientation module, $R^{\circ}$, is the left $R G$-module corresponding to the right module $H^{*}(G ; R G)$. In every known case, the action of $G$ on its orientation module has image contained in $\{ \pm 1\} \subseteq \operatorname{Aut}_{R}(R)$. We shall say that $G$ is standard if this holds. The question of whether every Poincaré duality group is standard is posed by Bestvina in $\S 2.2$ of [4]. Note that the topological definition of a Poincare duality group used in [4] is far more restrictive than the algebraic definition used in this paper, which is equivalent to the definition of Bieri $\left[6^{-8}\right]$. 
Lemma 4.4. Let $G$ be a standard, non-orientable $\mathrm{PD}^{n}$ group over $R$ for some even $n$, and let $H$ be the kernel of the orientation map. If $G$ is of type FPP over $R$, then $\operatorname{Ind}_{H}^{G}(E(H))=2 E(G)$.

Proof. For an $R G$-module $M$ admitting a finite resolution by finitely generated projective modules, we introduce the notation $[M]$ for the element of $K_{0}(R G)$ represented by the alternating sum of the modules in such a resolution. For example, when $G$ is of type $\mathrm{FP},[R]$ is defined and equal to $E(G)$.

Take a projective resolution for $R$ over $R H$, and apply the exact functor $R G \otimes_{R H}-$. Inspection of this long exact sequence shows that $\operatorname{Ind}_{H}^{G}(E(H))$ is equal to $[R G / H]$. By the hypotheses on $G$, there is a short exact sequence of $R G$-modules:

$$
0 \rightarrow R^{\circ} \rightarrow R G / H \rightarrow R \rightarrow 0 .
$$

It follows that $\operatorname{Ind}_{H}^{G}(E(H))=2 E(G)$ if and only if $\left[R^{\circ}\right]=E(G)$. This can be proved using the same trick as in the proof of Theorem 2.1. By Lemma 3.2 there is a resolution for $R$ over $R G$

$$
0 \rightarrow P_{n} \rightarrow P_{n-1} \rightarrow \cdots \rightarrow P_{1} \rightarrow P_{0} \rightarrow R \rightarrow 0,
$$

in which each $P_{i}$ is a projective permutation module. Applying the functor $(-)^{*}$ to this projective resolution gives rise to a long exact sequence

$$
0 \rightarrow P_{0}^{*} \rightarrow P_{1}^{*} \rightarrow \cdots \rightarrow P_{n-1}^{*} \rightarrow P_{n}^{*} \rightarrow R^{\circ} \rightarrow 0 .
$$

The claim follows by comparing sequences (4.1) and (4.2).

A polycyclic-by-finite group $G$ admits a proper, cocompact action on a contractible manifold (see, for example, the introduction to [29]). It follows that $G$ is of type FP over $R$ if and only if the order of every finite subgroup of $G$ is a unit in $R$. Moreover, in this case, $G$ is of type FPP and is a Poincare duality group over $R$, of dimension equal to that of the manifold. It also follows that $G$ is standard in the sense of Lemma 4.4.

Corollary 4.5. Let $J$ be polycyclic-by-finite, and let $k$ be a field over which $J$ is a non-orientable $\mathrm{PD}^{2 n}$ group. Let $H$ be the orientable subgroup of $J$, and let $G$ be the double of $J$ along $H$. Then $G$ is an orientable $\mathrm{PD}^{2 n+1}$ group over $k$, and $E(G) \in K_{0}(k G)$ has order two if and only if $E(J)$ is not in the image of $\iota: K_{0}(k H) \rightarrow K_{0}(k J)$.

Proof. A spectral sequence argument shows that the direct product of a $\mathrm{PD}^{m}$ group and a $\mathrm{PD}^{l}$ group is a $\mathrm{PD}^{m+l}$ group, with orientation module the tensor product (over $k$ ) of the orientation modules for the two factors [7, Proposition 9.16].

The first claim follows from the fact that $G$ is the orientable index-two subgroup of the product of $J$ and the infinite dihedral group. To see this, note that the infinite dihedral group $D$ is the free product $C_{2} * C_{2}$ of two cyclic groups of order two. Thus there is a decomposition of $J \times D$ as a free product with amalgamation:

$$
J \times D=J \times\left(C_{2} * C_{2}\right)=\left(J \times C_{2}\right) *_{J \times\{1\}}\left(J \times C_{2}\right) .
$$


For the orientation action of $\left(J \times C_{2}\right) *_{J}\left(J \times C_{2}\right)$ on $k$, the generators for the two copies of $C_{2}$ act non-trivially, and each of the copies of $J$ acts via the orientation action for $J$. Standard techniques for computing subgroups of graphs of groups [13, Chapter 1] show that the kernel of the orientation action is isomorphic to $J *_{H} J$ as required.

The second claim follows from Proposition 4.2 and Lemma 4.4.

To apply the above, a method for calculating $K_{0}$ for some polycyclic-by-finite groups is needed. The following theorem is from $[\mathbf{2 5}]$.

Theorem 4.6 (Moody). Let $G$ be polycyclic-by-finite, and let $\Phi(G)$ be the Frobenius category of $G$, as described in the proof of Corollary 3.3. Let $R$ be regular Noetherian and suppose that the order of every finite subgroup of $G$ is invertible in $R$. Then the natural map

$$
\iota: \operatorname{colim}_{H \in \Phi} K_{0}(R H) \rightarrow K_{0}(R G)
$$

is a surjection.

In fact, Moody proves a similar result for $G_{0}(R G)$ (the Grothendieck group of all finitely generated $R G$-modules) for any Noetherian $R$. The extra conditions that $R$ be regular and that the order of each finite subgroup of $G$ be invertible in $R$ allow one to state the result for $K_{0}$ instead, since they imply that $K_{0}(R G)$ is naturally isomorphic to $G_{0}(R G)$.

Quinn's Theorem $[\mathbf{2 9}]$ states that in the case when $R=\mathbb{Q}$, the map $\iota$ as above is an isomorphism. We shall not need to use this, however.

\section{The prism group}

In this section we study a single example, namely the group $G$ defined to be the orientable subgroup of the group generated by reflections in the sides of a right regular triangular prism in $\mathbb{R}^{3}$. For this $G$, and for any field $k$ of characteristic not equal to 2 or 3 , we compute $K_{0}(k G)$, and show that $E(G) \in K_{0}(k G)$ has order two if and only if $k$ does not contain a primitive cube root of unity.

Let $J$ be the $(3,3,3)$-triangle group, i.e. the group generated by the reflections in the sides of a regular triangle in the Euclidean plane [16, p. 89]. The group $J$ has the presentation

$$
J=\left\langle a, b, c \mid a^{2}, b^{2}, c^{2},(a b)^{3},(b c)^{3},(c a)^{3}\right\rangle .
$$

The group $G$ is the orientable subgroup of the product of $J$ and the infinite dihedral group. As in the proof of Corollary 4.5, $G$ may also be viewed as the double of $J$ along $H$, its orientable subgroup of index two. Before starting our calculations, we record some facts about $J$ and $H$.

The group $J$ is expressible as an extension with kernel $\mathbb{Z}^{2}$ and quotient the dihedral group $D_{3}$ of order six. $J$ has three conjugacy classes of maximal finite subgroup, each isomorphic to $D_{3}$. All elements of $J$ of order two are conjugate. Every element of $J$ of order three is conjugate to its inverse, and there are three conjugacy classes of subgroup of $J$ of order three, one contained in each conjugacy class of $D_{3}$. The group $H$ has three 
conjugacy classes of maximal finite subgroup, each cyclic of order three, and has six conjugacy classes of elements of order three. All of these facts may be verified easily by considering the action of $J$ on the Euclidean plane, bearing in mind that any finite subgroup $K$ of $J$ must have a fixed point (for example, the centroid of any $K$-orbit).

Lemma 5.1. Let $J, H$ and $k$ be as above, and let $\omega$ be a primitive cube root of unity in some extension of $k$. Then $K_{0}(k J) \cong \mathbb{Z}^{\oplus 5}$,

$$
\operatorname{coker}\left(\operatorname{Ind}_{H}^{J}\right)= \begin{cases}\mathbb{Z}, & \text { if } \omega \in k, \\ \mathbb{Z} \oplus\left(\mathbb{Z}_{2}\right)^{\oplus 3}, & \text { otherwise }\end{cases}
$$

and $E(J)$ is in the image of $\operatorname{Ind}_{H}^{J}$ if and only if $\omega \in k$.

Proof. First we compute the limit of $K_{0}(k-)$ over the Frobenius category of $J$. Since $J$ is abelian-by-finite, and all maximal finite subgroups of $J$ map isomorphically to a fixed finite quotient of $J$, this limit is easy to describe. It is isomorphic to the quotient of three copies of $K_{0}\left(k D_{3}\right)$ in which the three images of $\operatorname{Ind}_{C_{2}}^{D_{3}}$ have been identified. (Here $C_{2}$ denotes a subgroup of $D_{3}$ of order two.) Since the characteristic of $k$ is not equal to two or three, there are three irreducible $k D_{3}$-modules, the trivial module (denoted ' 1 '), a non-trivial one-dimensional module $\epsilon$, and a two-dimensional module $\alpha$. The image of the induction from $C_{2}$ is generated by $1+\alpha$ and $\epsilon+\alpha$. Listing the three $k D_{3}$-modules in the order $1, \epsilon, \alpha$ for $i=1,2,3$, the limit over the Frobenius category is $\mathbb{Z}^{\oplus 9}$ modulo the subgroup generated by the rows of matrix (5.1), in which blank entries are zeros omitted for clarity:

$$
\begin{array}{ccccccccc}
1 & 0 & 1 & & & & -1 & 0 & -1 \\
0 & 1 & 1 & & & & 0 & -1 & -1 \\
& & & 1 & 0 & 1 & -1 & 0 & -1 \\
& & & 0 & 1 & 1 & 0 & -1 & -1
\end{array}
$$

This is isomorphic to $\mathbb{Z}^{\oplus 5}$ as claimed.

By Moody's Theorem, quoted as Theorem 4.6, there is an epimorphism $\iota$ from the limit (as computed above) to $K_{0}(k J)$, and by Proposition 1.8 of [9], the kernel of $\iota$ is finite. Since in this case the limit is torsion free, it follows that Moody's map is an isomorphism.

Let $C_{3}$ denote a cyclic group of order three. If $\omega \notin k$, there are two irreducible $k C_{3^{-}}$ modules, the trivial module and a two-dimensional module. The images of these under $\operatorname{Ind}_{C_{3}}^{D_{3}}$ are $1+\epsilon$ and $2 \alpha$, respectively. On the other hand, if $\omega \in k$, there are three irreducible $k C_{3}$-modules, each of dimension one, and their images under $\operatorname{Ind}_{C_{3}}^{D_{3}}$ are $1+\epsilon, \alpha$, and $\alpha$, respectively.

Applying Moody's Theorem to $H$, the image of $\operatorname{Ind}_{H}^{J}$ is equal to the sum of the images of $\operatorname{Ind}_{K}^{J}$, where $K$ ranges over the maximal finite subgroups of $H$. Representing $K_{0}(k J)$ as the quotient of $\mathbb{Z}^{\oplus 9}$ by the rows of (5.1), the image of $\operatorname{Ind}_{H}^{J}$ is generated 
by

$$
\begin{array}{lllllllllll}
1 & 1 & 0 & & & & & & & \\
0 & 0 & 2 & & & & & & & * \\
& & & 1 & 1 & 0 & & & & \\
& & & 0 & 0 & 2 & & & & * \\
& & & & & & 1 & 1 & 0 & \\
& & & & & & 0 & 0 & 2 & *
\end{array}
$$

if $\omega \notin k$. In the case when $\omega \in k$, the matrix $\left(5.2^{\prime}\right)$ representing the image of $\operatorname{Ind}_{H}^{J}$ is similar to (5.2), except that the three rows of (5.2) marked with an asterisk are divided by two. The cokernel of $\operatorname{Ind}_{H}^{J}$ is isomorphic to the quotient of $\mathbb{Z}^{\oplus 9}$ by the subgroup generated by the rows of (5.1) and (5.2) if $\omega \notin k$ (respectively, (5.1) and (5.2') if $\omega \in k$ ). The claimed description of this cokernel follows.

The Euler class of $J$ may be computed by considering the chain complex for the $J$ equivariant tessellation of the plane by triangles. The triangular 2-cells are permuted freely, transitively. There are three orbits of 1-cell, each with stabilizer of order two, and there are three orbits of 0-cell, with stabilizers the three conjugacy classes of the $D_{3}$ subgroup. Hence the Euler class of $J$ is represented by the vector

$$
\begin{aligned}
(1,1,2,0,0,0,0,0,0)-3(1,0,1,0,0,0,0,0,0)+(1,0,0,1,0,0,1,0,0) & \\
& =(-1,1,-1,1,0,0,1,0,0) .
\end{aligned}
$$

It may be checked that this vector is in the subgroup generated by the rows of (5.1) and $\left(5.2^{\prime}\right)$ but not in the subgroup generated by the rows of (5.1) and (5.2).

Theorem 5.2. Let $J, H$ and $k$ be as in the introduction to this section, and let $\omega$ be a primitive cube root of unity in some extension of $k$. Let $l$ be the degree of $k[\omega]$ over $k$, and let $G$ be the double of $J$ along $H$. Then $G$ is an orientable $\mathrm{PD}^{3}$ group over $k$ of type FPP,

$$
K_{0}(k G) \cong \mathbb{Z}^{\oplus 6} \oplus\left(\mathbb{Z}_{l}\right)^{\oplus 3},
$$

and $E(G) \in K_{0}(k G)$ has order $l$. In particular, $G$ is FL over $k$ if and only if $l=1$.

Proof. This follows from Corollary 4.5 and Lemma 5.1.

One can also obtain some information for integral domains. Suppose $R$ is any integral domain in which 6 is a unit. Then $E(G) \in K_{0}(R G)$ is defined and has order at most two by Theorem 2.1. If $R$ contains a primitive cube root of unity, it may be shown that $E(G)=0$. On the other hand, if the field of fractions of $R$ does not contain a primitive cube root of unity, the order of $E(G)$ is two.

\section{Virtually free groups}

In this section we show how computations in the $K$-theory of coproducts can be used to construct virtually free groups with torsion in $K_{0}$ of their group algebras. Perhaps the most surprising examples are groups $G$ and field extensions $K \geqslant k$ such that the map 
from $K_{0}(k G)$ to $K_{0}(K G)$ has non-trivial kernel. Simpler such examples can be given that are not group algebras, so we discuss these first.

Suppose that $K$ is a separable field extension of $k$ of degree $n$. The action of $K$ on itself gives a $k$-algebra embedding of $K$ in the matrix ring $S=M_{n}(k)$. Now $K_{0}(K)$ is isomorphic to $\mathbb{Z}$, with generator the free module of rank 1 , and $K_{0}(S)$ is isomorphic to $\mathbb{Z}$, generated by the module of column vectors, $k^{n}$, so that the free module represents $n$ times a generator. Let $R$ be the coproduct of two copies of $S$, amalgamating $K$. The results of Bergman or Waldhausen, stated in a special case as Theorem 4.1, give an exact sequence

$$
K_{0}(K) \rightarrow K_{0}(S) \oplus K_{0}(S) \rightarrow K_{0}(R) \rightarrow\{0\} .
$$

It follows that $K_{0}(R)$ is isomorphic to $\mathbb{Z} \oplus \mathbb{Z}_{n}$. On the other hand, if $\bar{k}$ is the algebraic closure of $k$, then $\bar{k} \otimes_{k} K \cong \bar{k} \times \cdots \times \bar{k}=\bar{k}^{n}, \bar{k} \otimes_{k} S \cong M_{n}(\bar{k})$, and the image of any of the $n$ simple projective modules for $\bar{k}^{n}$ maps to a generator for $K_{0}\left(M_{n}(\bar{k})\right)$. Hence $K_{0}\left(\bar{k} \otimes_{k} R\right)$ is isomorphic to $\mathbb{Z}$, generated by the free module.

The ring $R$ is not a group algebra, but there is a case in which $R$ can be viewed as a skew group algebra: if $K$ is a Galois extension of $k$, with Galois group $G$, then the natural skew group algebra $K * G$ is isomorphic to $M_{n}(k)$ (see $[\mathbf{1 0}$, p. 590]). Hence $R$ may be viewed as the skew group algebra of the free product $G * G$ with coefficients $K$, where each factor acts in the natural way on $K$. In this case $\bar{k} \otimes_{k} R$ is the skew group algebra with coefficients $\bar{k}^{n}$, where each of the two copies of $G$ acts by freely permuting the $n$ copies of $\bar{k}$. The special case of this construction in which $k=\mathbb{R}$ and $K=\mathbb{C}$ will be discussed in detail in $\S 10$. The group algebras given below in Propositions 6.1 and 6.2 are modelled on these examples.

Proposition 6.1. Let $p$ and $q$ be distinct primes, with $q$ dividing $p-1$, and let $J$ be a non-abelian group of order $p q$. Let $G$ be the double of $J$ along $H$, the order $p$ subgroup of $G$. Then $K_{0}(\mathbb{Q} G)$ contains an element of order $q$, which maps to zero in $K_{0}(\mathbb{C} G)$.

Proof. The group $J$ has three irreducible rational representations: the trivial representation $T$ of dimension one, a $(q-1)$-dimensional representation $\alpha$, faithful on the order $q$ quotient of $J$, and a $(p-1)$-dimensional representation $\beta$. The regular representation of $J$ is equal to $T+\alpha+q \beta$. The image of induction from $H$ is generated by $T+\alpha$ and the regular representation, and so the cokernel of this map contains $q$-torsion. From Proposition 4.2 it follows that $K_{0}(\mathbb{Q} G)$ contains $q$-torsion. In fact, $K_{0}(\mathbb{Q} G) \cong \mathbb{Z}^{4} \oplus \mathbb{Z}_{q}$.

On the other hand, $J$ has $q$ distinct one-dimensional irreducible complex representations, each containing $H$ in its kernel, and $(p-1) / q$ distinct $q$-dimensional irreducible complex representations, each of which is induced up from $H$. It follows that the image of $K_{0}(\mathbb{C} H)$ in $K_{0}(\mathbb{C} J)$ is a direct summand, and hence $K_{0}(\mathbb{C} G)$ is free abelian (of rank $2 q-1+(p-1) / q)$.

We give more information in the special case of dihedral groups.

Proposition 6.2. Let $p$ be an odd prime, and let $G$ be the double of the dihedral group of order $2 p$ along its cyclic subgroup of order $p$. Let $k$ be a field of characteristic 
zero, and let $\zeta$ be a primitive pth root of 1 in some extension of $k$. Finally, define $m$ to be the degree of the field extension $m=\left|k\left[\zeta+\zeta^{-1}\right]: k\right|$, and let $r=(p-1) / 2 m$. Then

$$
K_{0}(k G) \cong \begin{cases}\mathbb{Z}^{\oplus r+3}, & \text { if } \zeta \in k\left[\zeta+\zeta^{-1}\right], \\ \mathbb{Z}^{\oplus r+3} \oplus\left(\mathbb{Z}_{2}\right)^{\oplus r}, & \text { otherwise. }\end{cases}
$$

Proof. Define $l$ by the equation $l=|k[\zeta]: k|$. The irreducible $k$-representations of $C_{p}$ are the trivial representation of dimension one, and $(p-1) / l$ others of dimension $l$. The irreducible $k$-representations of $D_{p}$ are the trivial representation, the orientation representation of dimension one, and $r=(p-1) / 2 m$ others of dimension $2 m$. In the case when $l=m$, or equivalently when $\zeta \in k\left[\zeta+\zeta^{-1}\right]$, the image of induction from $C_{p}$ to $D_{p}$ is spanned by the sum of the two one-dimensional representations and the other representations. Hence in this case the cokernel of induction is infinite cyclic. In the case when $l=2 m$, each non-trivial irreducible representation of $C_{p}$ induces up to two copies of one of the irreducible representations of $D_{p}$, and the cokernel of induction is infinite cyclic direct sum $r$ copies of $\mathbb{Z}_{2}$. The claims follow from Proposition 4.2.

Proposition 6.3. Let $p$ be a prime and let $J$ be a direct product of $m+1$ cyclic groups of order $p$. Let $H_{1}, \ldots, H_{n}$ be the order $p$ subgroups of $J$, so that $n=\left(p^{m+1}-1\right) /(p-1)$. Let $G$ be the double of $J$ along $H_{1}, \ldots, H_{n}$. For any field $k$ of characteristic not equal to $p, K_{0}(k G)$ contains elements of order $p^{m}$.

Proof. As usual, we use the description of $K_{0}(k G)$ given by Proposition 4.2. For $i=$ $1, \ldots, n$, let $T_{i}$ (respectively, $R_{i}$ ) denote the trivial (respectively, regular) representation of $H_{i}$, and similarly let $T_{0}$ (respectively, $R_{0}$ ) denote the trivial (respectively, regular) representation of $J$. By hypothesis each $T_{i}$ is projective. By symmetry, there are integers $r$ and $s$ such that

$$
\iota\left(\sum_{i=1}^{n} T_{i}\right)=r T_{0}+s R_{0}
$$

where $\iota$ is the direct sum of the induction maps as in the statement of Proposition 4.2. More formally, the automorphism group of $J$, which is isomorphic to the general linear group $G L(m+1, p)$, acts on the system consisting of $J$ and the subgroups $H_{1}, \ldots, H_{n}$, permuting the $H_{i}$. For this action,

$$
\iota: \bigoplus_{i=1}^{n} K_{0}\left(k H_{i}\right) \rightarrow K_{0}(k J)
$$

is an $\operatorname{Aut}(J)$-equivariant map. The left-hand side of (6.1) is fixed by the action of $\operatorname{Aut}(J)$, and hence so is its image under $\iota$. This image must be as described in the right-hand side of $(6.1)$, since the fixed subgroup of $K_{0}(k J)$ for the action of $\operatorname{Aut}(J)$ is generated by the trivial representation and the regular representation.

A comparison of $k$ dimensions and numbers of trivial summands in (6.1) shows that $r=p^{m}$ and $s=\left(p^{m}-1\right) /(p-1)$. Since $\iota\left(R_{1}\right)=R_{0}$, it follows that $p^{m} T_{0}$ is in the image of $\iota$. On the other hand, the index of each $H_{j}$ in $J$ is $p^{m}$, so any $k J$-module in the image 
of $\iota$ has dimension divisible by $p^{m}$. It follows that the subgroup of coker $(\iota)$ generated by $T_{0}$ is cyclic of order $p^{m}$.

Corollary 6.4. For any positive integer $n$ and any field $k$ of characteristic coprime to $n$, there is a virtually free group $G$ such that $K_{0}(k G)$ contains elements of order $n$.

Proof. Take the free product of examples as in Proposition 6.3 for the distinct prime powers dividing $n$.

These examples answer a question posed in $\S 2.5$ of $[\mathbf{9}]$, as do certain examples in Moselle's thesis [26]. Lemma 2.2 of [9] shows that our methods will never find $p$-torsion in $K_{0}(k G)$ for $k$ of characteristic $p$.

\section{Poincaré duality groups}

In this section we combine results of previous sections to exhibit more examples of Poincaré duality groups over the rationals that are FL over the complex numbers but not FL over the rationals. Our first such example is the index-two orientable subgroup of the direct product of a $(p, p, p)$-triangle group and an infinite dihedral group, for $p \geqslant 5$ a prime. The calculations are very similar to those in $\S 5$, except that we cannot fully determine $K_{0}$, since no analogue of Moody's Theorem (Theorem 4.6) is known.

Higher-dimensional examples could be constructed using similar techniques. It is, however, easier to apply the 'Suspension Lemma', given below as Corollary 7.3, to obtain higher-dimensional examples from those constructed already.

Let $J$ be the $(p, p, p)$-triangle group, that is, the group generated by reflections in the sides of a hyperbolic triangle with all angles $2 \pi / p$. Arguments similar to those outlined in $\S 5$ show that $J$ has three conjugacy classes of maximal finite subgroup, each dihedral of order $2 p$, three conjugacy classes of cyclic subgroup of order $p$ (one in each class of dihedral group), and one conjugacy class of elements of order two. Let $D(i)$ and $C(i)$, for $i=1,2,3$, be representatives of the three conjugacy classes of $D_{p}$ subgroups and $C_{p}$ subgroups, respectively. There is a homomorphism $\pi$ from $J$ to $D_{p}$ that restricts to an isomorphism on each $D(i)$, and hence has torsion-free kernel. (Just send the three generators of $J$ of order two to any three distinct elements of $D_{p}$ of order two.) The kernel of any such $\pi$ is the fundamental group of a closed orientable surface of Euler characteristic $3-p$. The image of the orientable index-two subgroup $H$ of $J$ under $\pi$ is $C_{p} \leqslant D_{p}$.

Theorem 7.1. Let $k$ be a field of characteristic zero, let $\zeta$ be a primitive $p$ th root of 1 in some extension of $k$, let $H, J$ be as described above, and let $G$ be the double of $J$ along $H$. Then $G$ is an orientable $\mathrm{PD}^{3}$ group over $k$, and $E(G) \in K_{0}(k G)$ has order two if $\zeta \notin k\left[\zeta+\zeta^{-1}\right]$, and is equal to zero otherwise.

Proof. The group $G$ is the orientable index-two subgroup of $J \times D_{\infty}$, so is an orientable $\mathrm{PD}^{3}$ group of type FPP over $k$. It follows from Theorem 2.1 that $E(G)$ has order at most two. 
Let $\epsilon$ denote the non-trivial one-dimensional representation of $D_{p}$, and define a representation $\alpha$ of $D_{p}$ by the equation $k D_{p}=1+\epsilon+2 \alpha$. Other equivalent descriptions of $\alpha$ are that $\alpha$ consists of a direct sum of one copy of each irreducible $k$-representation of $D_{p}$ of dimension greater than one, or that $k D_{p} / C_{2}=1+\alpha$. Arguments similar to those used in the proof of Proposition 6.2 show that $\alpha$ is in the image of induction from $k C_{p}$ if and only if $\zeta \in k\left[\zeta+\zeta^{-1}\right]$.

The natural $J$-equivariant triangulation of the hyperbolic plane has one free orbit of 2 -simplices, three orbits of edges with stabilizer of order two, and three orbits of vertices with stabilizers conjugate to $D(1), D(2)$ and $D(3)$, respectively. It follows that the Euler class of $J$ is

$$
\begin{aligned}
E(J) & =[k J]-3\left[k J / C_{2}\right]+\sum_{i=1}^{3}[k J / D(i)] \\
& =[k J]-\sum_{i=1}^{3}\left[\operatorname{Ind}_{D(i)}^{J}(\alpha)\right] .
\end{aligned}
$$

If $\zeta \in k\left[\zeta+\zeta^{-1}\right]$, there is a $k$-representation $\xi$ of $C_{p}$ such that $\operatorname{Ind}_{C_{p}}^{D_{p}}(\xi)=\alpha$. Hence $E(J)$ is in the image of induction from $H$, because

$$
E(J)=\operatorname{Ind}_{H}^{J}\left([k H]-\sum_{i=1}^{3}\left[\operatorname{Ind}_{C(i)}^{H}(\xi)\right]\right) .
$$

By Proposition 4.3 and Lemma 4.4, it follows that in this case $E(G)=0$.

For the converse, note that the image of $E(J)$ under the homomorphism $\pi: J \rightarrow D_{p}$ is

$$
\left[k D_{p}\right]-3 \alpha=1+\epsilon-\alpha .
$$

If $\zeta \notin k\left[\zeta+\zeta^{-1}\right]$, then this is a non-zero element in the cokernel of the induction from $C_{p}$ to $D_{p}$. From the description of $E(G)$ and $K_{0}(k G)$ given in Proposition 4.2, it follows that in this case $E(G)$ is non-zero.

To exhibit higher-dimensional Poincaré duality groups that are FL over the complex numbers but not FL over the rational numbers we shall make use of a product formula given below in Theorem 7.2. Before stating this theorem, we make another definition.

Suppose that $G$ is of type FP over a $\operatorname{ring} R$, that $P_{*}$ is a finite projective resolution of $R$ over $R G$, and that $\phi: R \rightarrow k$ is a homomorphism from $R$ to a field. In this case define $\chi_{\phi}(G)$ as

$$
\chi_{\phi}(G)=\sum_{i \geqslant 0}(-1)^{i} \operatorname{dim}_{k} \operatorname{Hom}_{R G}\left(P_{i}, k\right)=\sum_{i \geqslant 0}(-1)^{i} \operatorname{dim}_{k} H^{i}(G ; k) .
$$

If $P$ is a projective permutation module with $R$-basis a $G$-set $\Omega$, the $k$-dimension of $\operatorname{Hom}_{R G}(P, k)$ is equal to the number of $G$-orbits in $\Omega$. In particular, in the case when $G$ is FPP over $R$, the integer $\chi_{\phi}(G)$ is independent of the choice of $k$ and $\phi$, and will be denoted $\chi(G)$. Geometrically, if $G$ acts cocompactly on an $R$-acyclic simplicial complex 
$X$ with stabilizers of order invertible in $R$, then $\chi(G)$ is equal to the Euler characteristic of the quotient space $X / G$.

Theorem 7.2. Fix a (commutative, unital) ring $R$, let $G=H \times K$, and suppose that $H$ and $K$ are FP (over $R$ ).

(a) There is a bilinear product $K_{0}(R H) \times K_{0}(R K) \rightarrow K_{0}(R G)$ defined on generators by $[P] \cdot[Q]=\left[P \otimes_{R} Q\right]$.

(b) $G$ is $\mathrm{FP}$, and $E(G)=E(H) \cdot E(K)$.

(c) If $K$ is $\mathrm{FPP}$ and $\chi(K)= \pm 1$, then $E(G)=0$ if and only if $E(H)=0$.

(d) If $K$ is FL and $\chi(K)= \pm 1$, then $G$ is FL if and only if $H$ is FL.

Proof. Throughout this proof the symbol ' $\otimes$ ' will indicate a tensor product over $R$. It is well known (and easily verified) that the maps

$$
r(h \otimes k) \mapsto r(h k), \quad r g \mapsto r\left(\pi_{1}(g) \otimes \pi_{2}(g)\right)
$$

define inverse isomorphisms between the $R$-algebras $R H \otimes R K$ and $R G$, where $\pi_{1}$ (respectively, $\pi_{2}$ ) denotes the projection from $G$ onto $H$ (respectively, $K$ ). Hence for any $R H$ module $M$ and $R K$-module $N$, there is a natural $R G$-module structure on $M \otimes N$. In the case when $M$ is free of rank $m$ and $N$ is free of rank $n, M \otimes N$ is free of rank $m n$. If $P$ is a direct summand of $M$ and $Q$ is a direct summand of $N$, then $P \otimes Q$ is a direct summand of $M \otimes N$. It follows that the tensor product of a projective $R H$-module and a projective $R K$-module is a projective $R G$-module. The fact that the formula $[P] \cdot[Q]=[P \otimes Q]$ gives a well-defined bilinear product $K_{0}(R H) \times K_{0}(R K) \rightarrow K_{0}(R G)$ follows from the additivity properties of the tensor product.

The underlying $R$-module for a projective $R H$-module or $R K$-module is necessarily $R$-projective. Hence if $P_{*}$ (respectively, $Q_{*}$ ) is a projective resolution for $R$ over $R H$ (respectively, $R K$ ), the Künneth formula may be applied to deduce that the total complex for the double complex $P_{*} \otimes Q_{*}$ is a resolution for $R$ over $R G$. Since we have already shown that each $P_{i} \otimes Q_{j}$ is a projective $R G$-module (finitely generated whenever $P_{i}$ and $Q_{j}$ are), claim (b) follows.

If $E(H)=0$, then $E(G)=0$ by (b). For the converse, note that the projection $\pi_{1}: G \rightarrow H$ induces a map $\pi_{*}: K_{0}(R G) \rightarrow K_{0}(R H)$. Letting $K^{\prime}$ denote a subgroup of $K$ whose order is invertible in $R$, one sees that for any projective $R H$-module $P$, $\pi_{*}\left([P] \cdot\left[R K / K^{\prime}\right]\right)=[P]$. It follows that when $K$ is FPP, $\pi_{*}([P] \cdot E(K))=\chi(K)[P]$, and so $\pi_{*}(E(G))=\pi_{*}(E(H) \cdot E(K))=\chi(K) E(H)$. Hence when $\chi(K)= \pm 1, E(H)=$ $\pm \pi_{*}(E(G))$ and claim (c) follows.

If $H$ and $K$ are both FL, then (b) implies that $G$ is FL. As in the previous paragraph, the hypotheses that $K$ is FPP and $\chi(K)= \pm 1$ imply that $E(H)= \pm \pi_{*}(E(G))$. Since also $\pi_{*}([R G])=[R H]$, claim (d) follows.

See $[\mathbf{2 4}]$ for a formula similar to Theorem 7.2 (b) and [15] for a result close to the case $R=\mathbb{Z}$ of Theorem $7.2(\mathrm{~d})$. 
Corollary 7.3. Let $G$ be a group of type FP over $R$, let $D$ denote the infinite dihedral group and let $H$ denote either the free group of rank two or the fundamental group of a closed (non-orientable) surface of Euler characteristic - 1 .

(a) $G \times H$ is FP over $R$. Moreover, $E(G \times H)=0$ if and only if $E(G)=0$, and $G \times H$ is $\mathrm{FL}$ over $R$ if and only if $G$ is.

(b) If 2 is a unit in $R$, then $G \times D$ is FP over $R$. Moreover, $E(G \times D)=0$ if and only if $E(G)=0$, and if $G \times D$ is FL over $R$, then so is $G$.

Only the case of Corollary 7.3 involving the infinite dihedral group is needed below, but we draw attention to the other two cases for the following reason. The other groups have the advantages that they are torsion free, so no restriction on $R$ is needed, and that they are FL over any $R$. (Since the dihedral group $D$ is not FL over any (non-trivial) ring, it can happen that $G$ is FL over $R$, but $G \times D$ is not.) On the other hand, the product of $G$ and the free group of rank two cannot be a Poincaré duality group. The fundamental group, $H$, of a surface of Euler characteristic -1 does not have this defect, but of course taking a direct product with $H$ increases cohomological dimension by two.

Proof of Corollary 2.4. In the case when $n=3$, Theorems 2.2, 5.2 and 7.1 exhibit groups $G$ that are $\mathrm{PD}^{n}$ groups over $\mathbb{Q}$ and also have the properties that $E(G)=0$ in $K_{0}(\mathbb{C} G)$ but $\tilde{E}(G) \neq 0$ in $K_{0}(\mathbb{Q} G)$. If $G$ is an example of an $n$-dimensional group having these properties, Corollary 7.3 implies that the product of $G$ and an infinite dihedral group is an example of dimension $n+1$. Any such group is FL over $\mathbb{C}$ but not FL over $\mathbb{Q}$.

\section{More virtually free groups}

The Euler class of a virtually free group can never be a non-zero torsion element, as shown below in Proposition 8.1. It can, however, happen that the Wall finiteness obstruction $\tilde{E}(G)$ for a virtually free group $G$ is a torsion element in $\tilde{K}_{0}(R G)$, the quotient of $K_{0}(R G)$ by the subgroup generated by $[R G]$. One example of this appeared (unremarked) earlier in the paper: the case $p=n=2$ of Proposition 6.3. In this section, such examples are exhibited using Theorem 8.2, a relative version of Theorem 2.1.

Proposition 8.1. Let $G$ be a (finitely generated) virtually free group and $R$ a ring over which $G$ is FP. Then $E(G) \in K_{0}(R G)$ is either zero or has infinite order.

Proof. The Euler class of a non-cyclic free group has infinite order. It follows that if $G$ has a non-cyclic free subgroup, then $E(G)$ has infinite order, and it remains to consider the virtually cyclic case. If $G$ has an infinite cyclic subgroup of finite index, then either $G=N: \mathbb{Z}$, or $G=A *_{N} B$, where $N$ is a finite normal subgroup, and in the second case $|A: N|=|B: N|=2[\mathbf{1 3}$, IV.6.12]. These two cases correspond to actions on a line with one orbit of edges and one or two orbits of vertices, respectively. In the first case the edge and vertex stabilizers are both equal to $N$, so $E(G)=[R G / N]-[R G / N]=0$. In the second case, $E(G)=[R G / A]+[R G / B]-[R G / N]$, and has infinite order, since its image under the natural map from $K_{0}(R G)$ to $K_{0}(R)$ is equal to $[R]$. 
Theorem 8.2. Let $X$ be a contractible triangulated $n$-manifold with boundary for some odd $n$, and suppose that $G$ acts on $X$, preserving the orientation of $X$, with finitely many orbits of simplices, freely on the boundary of $X$, and with stabilizers of order invertible in $R$. Then $G$ is FPP over $R$, and the Wall obstruction $\tilde{E}(G) \in \tilde{K}_{0}(R G)$ has order at most two.

Proof. Replacing $X$ by its barycentric subdivision, we may suppose that the simplicial chain complex $C_{*}=C_{*}(X ; R)$ (with $R$-basis the simplices of $X$ ) is a sum of permutation modules $R G / H_{j}$ where each $\left|H_{j}\right|$ is a unit in $R$. Since $X$ is contractible, $C_{*}$ is an $R G$ projective resolution for $R, G$ is FPP over $R$, and

$$
E(G)=\sum_{i}(-1)^{i}\left[C_{i}\right] \in K_{0}(R G)
$$

Let $D^{*}$ be the cochain complex with compact supports for $X$ relative to its boundary $\delta X$, so that $D^{*}$ has $R$-basis the characteristic functions of those simplices of $X$ that are not contained in $\delta X$. Since $G$ acts freely on $\delta X$, for each $i$, there is a finitely generated free $R G$-module $F_{i}$ such that $D^{i} \oplus F_{i} \cong C_{i}$. By Poincaré duality for $X$ (see [27]), there is a long exact sequence

$$
0 \rightarrow D^{0} \rightarrow D^{1} \rightarrow \cdots \rightarrow D^{n-1} \rightarrow D^{n} \rightarrow R \rightarrow 0 .
$$

It follows that

$$
E(G)=\sum_{i}(-1)^{n-i}\left[D^{i}\right]=(-1)^{n} \sum_{i}(-1)^{i}\left[C_{i}\right]+(-1)^{n+1} \sum_{i}(-1)^{i}\left[F_{i}\right] .
$$

Since $n$ is odd, adding equations (8.1) and (8.2) gives

$$
2 E(G)=(-1)^{n+1} \sum_{i}(-1)^{i}\left[F_{i}\right]
$$

and hence the claim.

Let $J$ be a non-cyclic finite subgroup of the rotation group $S O(3)$. Recall that the natural action of $J$ on the 2-sphere has three non-free orbits. Let $H_{1}, H_{2}$ and $H_{3}$ be stabilizers of points in these three orbits.

Corollary 8.3. For $J, H_{i}$ as above, let $G$ be the double of $J$ along the $H_{1}, H_{2}, H_{3}$, and let $R$ be a ring in which $|J|$ is a unit. Then $G$ is a virtually free group of type FPP over $R$ and the Wall obstruction $\tilde{E}(G)$ in $\tilde{K}_{0}(R G)$ has order at most two.

Proof. We shall build a contractible 3-manifold with a $G$-action satisfying the conditions of Theorem 8.2. View the 3 -sphere as the 1-point compactification of $\mathbb{R}^{3}$. The usual $S O(3)$ action on $\mathbb{R}^{3}$ extends to an action on the 3 -sphere with fixed points at 0 and $\infty$. Let $B_{1}$ be the closed unit ball in $\mathbb{R}^{3}$ centred at 0 , and let $B_{2}$ be the complement in the 3 -sphere of the open ball of radius two, so that $B_{2}$ is a ball centred at $\infty$. Pick points $x_{i}$ for $i=1,2,3$ on the surface of $B_{1}$ in distinct $J$-orbits, such that the stabilizer of $x_{i}$ is 
$H_{i}$. Finally, for each $i$, let $C_{i}$ be a cylinder joining $B_{1}$ and $B_{2}$ with $x_{i}$ on its axis. Pick a $J$-equivariant triangulation for the union of $B_{1}, B_{2}$ and the images under $J$ of the $C_{i}$. (The radius of $C_{i}$ should be sufficiently small that these images are disjoint.)

This gives $J$-spaces $B_{j}$ for $j=1,2$ and for each $i$ an $H_{i}$-space $C_{i}$, together with $H_{i^{-}}$ equivariant attaching maps from one end of $C_{i}$ to the boundary of $B_{j}$ for each $i$ and $j$. Moreover, the singular set of $B_{j}$ (which is the central point together with a collection of intervals meeting at the centre) is a $J$-equivariant retract of $B_{j}$, the singular set of $C_{i}$ (which is its central line) is an $H_{i}$-equivariant retract of $C_{i}$, and these retractions can be chosen to be compatible with the gluing of $C_{i}$ to $B_{j}$. Now view $B_{j}$ as a $J_{j}$-space for $j=1,2$.

Each of the five spaces constructed so far can be 'induced up to $G$ ', to give $G$-spaces $G \times_{J_{j}} B_{j}$ and $G \times_{H_{i}} C_{i}$, and the $H_{i}$-equivariant attaching maps give rise to $G$-equivariant attaching maps between these five induced $G$-spaces. The resulting $G$-space $X$ has a $G$ tree as an equivariant retract (the tree corresponding to the given expression for $G$ as a graph of groups), and so $X$ is contractible. Moreover, $X$ is a 3 -manifold with boundary, and $G$ acts freely on $\delta X$. Hence Theorem 8.2 applies to $X$, and the claim follows.

The next proposition completes the proof of Theorem 2.3.

Proposition 8.4. Let $J$ be a finite subgroup of $S O(3)$, and construct $G$ as in Corollary 8.3. If $J$ is $D_{n}$ for $n$ even, or $A_{4}, S_{4}$ or $A_{5}$, then $\tilde{E}(G) \in \tilde{K}_{0}(\mathbb{C} G)$ has order two. If $J=D_{n}$ for $n$ odd, then $\tilde{E}(G) \in \tilde{K}_{0}(\mathbb{C} G)$ is zero, but $\tilde{E}(G) \in \tilde{K}_{0}(\mathbb{Q} G)$ has order two.

Proof. By Corollary 8.3, the order of $\tilde{E}(G)$ is at most two. Since $\tilde{K}_{0}(k J)$ is free abelian, it follows from Proposition 4.2 that $\tilde{E}(G)=0 \in \tilde{K}_{0}(k G)$ if and only if the trivial $k J$-module is a sum of induced modules from the $H_{i}$. If $J$ is the symmetry group of a platonic solid, or $D_{n}$ for $n$ even, then each of the $H_{i}$ has even index in $J$. Thus in this case any induced module has even $k$-dimension, so the trivial module is not a sum of induced modules.

If $J=D_{n}$ for $n$ odd, the trivial $\mathbb{C} C_{2}$-module induces up to $J$ as the sum of the trivial $\mathbb{C} J$-module direct sum one copy of each irreducible $\mathbb{C} J$-module of dimension two. Since each irreducible two-dimensional $\mathbb{C} J$-module is induced up from $C_{n}$, it follows that the trivial $\mathbb{C} J$-module is a sum of induced modules as required.

It remains to check that when $J=D_{n}$ for some odd $n$, the trivial $\mathbb{Q} J$-module is not a sum of induced modules (from proper subgroups). In the case when $n$ is an odd prime $p$, there are only three irreducible $\mathbb{Q} J$-modules, and it can be checked that induced modules (from any proper subgroup) consist of an even number of $\mathbb{Q} J$-irreducibles. This proves the claim when $n=p$. The claim for general odd $n$ follows, since if $p$ divides $n$, there is a surjective homomorphism from $D_{n}$ to $D_{p}$, which takes the trivial $\mathbb{Q} D_{n}$-module to the trivial $\mathbb{Q} D_{p}$-module.

\section{Comparing FL and FH}

Bestvina and Brady define $G$ to be of type FH over $R$ if there is an $R$-acyclic, $G$-free $G$ CW-complex $Y$ with finitely many orbits of cells [5]. Using the simplicial approximation 
theorem it may be shown that any $G$-CW-complex $Y$ is $G$-homotopy equivalent to a simplicial complex $X$ on which $G$ acts simplicially. Moreover, if $Y$ has finitely many orbits of cells, $X$ has finitely many orbits of simplices. Thus there is no loss of generality if one assumes that the space $Y$ is a simplicial complex.

If $G$ is $\mathrm{FH}$ over $R$, the chain complex for the space $Y$ is an $R G$-free resolution for the trivial module $R$, and hence $G$ is FL over $R$. The converse does not hold for all choices of $G$ and $R$, however, as we shall see below.

If $K$ is an extension field of a field $k$, then for any space $X$, and any $i$, the homology group $H_{i}(X ; K)$ is naturally isomorphic to $H_{i}(X ; k) \otimes K$. In particular, $X$ is $k$-acyclic if and only if $X$ is $K$-acyclic. Thus one obtains the following proposition.

Proposition 9.1. Let $K$ be an extension field of $k$. A group $G$ is $\mathrm{FH}$ over $K$ if and only if $G$ is $\mathrm{FH}$ over $k$.

Corollary 9.2. There are virtually free groups and $\mathrm{PD}^{3}$ groups over the complex numbers $\mathbb{C}$ that are FL over $\mathbb{C}$ but not $\mathrm{FH}$ over $\mathbb{C}$.

Proof. By Proposition 9.1, any group that is FL over $\mathbb{C}$ but is not FL over $\mathbb{Q}$ cannot be $\mathrm{FH}$ over $\mathbb{C}$. Virtually free groups having these properties are given in Proposition 8.4 and $\mathrm{PD}^{3}$ groups having these properties in Theorems 5.2 and 7.1.

To demonstrate that this corollary is interesting, we ought to show that many groups of type FL are also of type FH. This is the content of Theorem 9.4.

A CW-complex is said to be of finite type if it has finitely many cells in each dimension. (Note that a simplicial complex with finitely many vertices must be finite, so for this finiteness condition one really must consider $\mathrm{CW}$-complexes instead.) An EilenbergMac Lane space for a group $G$, denoted $K(G, 1)$, is a connected CW-complex whose fundamental group is $G$ and whose universal cover is contractible. The proposition below shows that every group considered in this paper has a finite-type Eilenberg-Mac Lane space.

Proposition 9.3. If $G$ is virtually torsion free and acts with finite stabilizers and finitely many orbits on a contractible simplicial complex $X$, then $G$ has a finite-type Eilenberg-Mac Lane space.

Proof. Let $H$ be a torsion-free finite-index normal subgroup of $G$, and let $Y$ be the universal cover of a finite-type Eilenberg-Mac Lane space for $G / H$. (The 'bar construction' shows that any finite group has a finite type Eilenberg-Mac Lane space [8].) Replacing $X$ by its barycentric subdivision if necessary, it may be assumed that the stabilizer of every simplex of $X$ fixes that simplex pointwise. Now $X$ may be viewed as a $G$-CW-complex whose cells just happen to be simplex shaped. Also $X \times Y$ is a contractible $G$-CW-complex with finitely many orbits of cells of each dimension. Since the stabilizer of any cell of $Y$ is $H$, and $H$ acts freely on $X$, it follows that $G$ acts freely on $X \times Y$, and the quotient $(X \times Y) / G$ is a finite-type $K(G, 1)$. 
Theorem 9.4. Let $R$ be a subring of the rationals, and let $G$ be a group with an Eilenberg-Mac Lane space of finite type. Then $G$ is FL over $R$ if and only if $G$ is FH over $R$.

Proof. Without any conditions on $G$ and $R, \mathrm{FH} \Rightarrow \mathrm{FL}$, but we must prove the converse. Let $Y$ be the universal cover of a finite-type Eilenberg-Mac Lane space for $G$. Let $n$ be the maximum of three and the cohomological dimension of $G$ over $R$, and let $X^{\prime}$ be the $(n-1)$-skeleton of $Y$. The $R$-chain complex $C_{*}^{\prime}$ for $X^{\prime}$ is the start of a finite $R G$-free resolution for $R$. Hence

$$
H^{\prime}=H_{n-1}\left(X^{\prime} ; R\right)=\operatorname{ker}\left(C_{n-1}^{\prime} \rightarrow C_{n-2}^{\prime}\right)
$$

is a stably free $R G$-module, i.e. there exists $m$ such that $H^{\prime} \oplus R G^{\oplus m}$ is free. Build $X^{\prime \prime}$ by attaching $m$ free $G$-orbits of $(n-1)$-spheres to $X^{\prime}$. (Equivalently, one could attach a bouquet of $m$ copies of the $(n-1)$-sphere to $Y / G$, and define $X^{\prime \prime}$ to be the $(n-1)$-skeleton of the universal cover of this space.) Now $X^{\prime \prime}$ is an $(n-1)$-dimensional $G$-free $G$-CWcomplex which is $(n-2)$-connected, and has the property that $H^{\prime \prime}=H_{n-1}\left(X^{\prime \prime} ; R\right)$ is a free $R G$-module, with $R G$-basis $h_{1}, \ldots, h_{l}$ say.

$H^{\prime \prime}$ is a submodule of the $(n-1)$-chains on $X^{\prime \prime}$, so each $h_{i}$ may be represented as a finite sum $h_{i}=\sum_{j} r_{i j} c_{j}$ for $r_{i j} \in R$ and $c_{j}$ an $(n-1)$-cell of $X^{\prime \prime}$. Since $R$ is a subring of $\mathbb{Q}$, there is an integer $s_{i}$ which is a unit in $R$ and such that for each $j, s_{i} r_{i j} \in \mathbb{Z}$. (Take $s_{i}$ to be the lowest common multiple of the denominators of the $r_{i j}$ when expressed as quotients of coprime integers.) The elements $s_{i} h_{i}$ form an $R G$-basis for $H^{\prime \prime}$, and each $s_{i} h_{i}$ lies in the integral $(n-1)$-chains on $X^{\prime \prime}$. Since $X^{\prime \prime}$ is $(n-2)$-connected, there is a map $f_{i}$ from the $(n-1)$-sphere to $X^{\prime \prime}$ sending a generator for the top homology group of the sphere to $s_{i} h_{i}$. Build $X$ by attaching $l$ free $G$-orbits of $n$-cells to $X^{\prime \prime}$ using the $f_{i}$ as attaching maps for a set of orbit representatives. This $X$ is the required $R$-acyclic $G$-free G-CW-complex.

\section{Detecting $K_{0}$}

In this section we give a proof that certain elements of $K_{0}$ have order two, that does not depend on $[\mathbf{2}, \mathbf{1 2}, \mathbf{3 1}, \mathbf{3 2}]$. This gives a self-contained and comparatively elementary proof of parts (a) and (b) of Theorem 2.3, together with some cases of Theorem 2.2. A different account of this argument has appeared in $[\mathbf{1 9}]$, so we omit some of the calculations.

Let $f$ be the embedding of $\mathbb{C}$ in $M_{2}(\mathbb{R})$ defined by

$$
f: r e^{i \theta} \mapsto\left(\begin{array}{cc}
r \cos \theta & -r \sin \theta \\
r \sin \theta & r \cos \theta
\end{array}\right) .
$$

Let $S_{1}$ and $S_{2}$ be copies of $M_{2}(\mathbb{R})$, and let $R$ be the coproduct of $S_{1}$ and $S_{2}$, amalgamating the images of $\mathbb{C}$. As a $\mathbb{C}$-bimodule, $M_{2}(\mathbb{R})$ is freely generated by

$$
I=\left(\begin{array}{ll}
1 & 0 \\
0 & 1
\end{array}\right) \quad \text { and } \quad A=\left(\begin{array}{ll}
0 & 1 \\
1 & 0
\end{array}\right)
$$


It follows that $R$ has $\mathbb{C}$-basis the alternating words in $A$ and $A^{\prime}$, the copies of $A$ lying in $S_{1}$ and $S_{2}$, respectively. Thus $R$ may be identified with the skew group ring $\mathbb{C} * D$, where $D$ is an infinite dihedral group generated by $A$ and $A^{\prime}$, and $A \lambda A=A^{\prime} \lambda A^{\prime}=\lambda^{*}$, the complex conjugate of $\lambda$, for each $\lambda \in \mathbb{C}$.

The map

$$
1 \otimes f: \mathbb{C} \otimes \mathbb{C} \rightarrow \mathbb{C} \otimes M_{2}(\mathbb{R})
$$

where all tensor products are over $\mathbb{R}$, is the inclusion of a subring isomorphic to $\mathbb{C} \times \mathbb{C}$ in $M_{2}(\mathbb{C})$. Hence $\mathbb{C} \otimes R$ may be identified with the coproduct of two copies of $M_{2}(\mathbb{C})$, amalgamating a subring isomorphic to $\mathbb{C} \times \mathbb{C}$. In terms of the description of $R$ as a skew group algebra, $\mathbb{C} \otimes R$ is isomorphic to $(\mathbb{C} \times \mathbb{C}) * D$, where $A(\lambda, \mu) A=A^{\prime}(\lambda, \mu) A^{\prime}=(\mu, \lambda)$ for any $(\lambda, \mu) \in \mathbb{C} \times \mathbb{C}$. The $\mathbb{R}$-algebra $R$ is one of the examples mentioned at the start of $\S 6$.

Now let $e$ be a rank-one idempotent in $M_{2}(\mathbb{R})$, and let $e, e^{\prime}$ denote copies of $e$ in $S_{1}$ and $S_{2}$, respectively. Let $[e]$ and $\left[e^{\prime}\right]$ denote the corresponding elements of $K_{0}(R)$. In $K_{0}\left(M_{2}(\mathbb{R})\right), 2[e]$ is equal to the free module of rank one. It follows that $2\left([e]-\left[e^{\prime}\right]\right)=$ $[R]-[R]=0$ in $K_{0}(R)$.

On the other hand, the image of $1 \otimes f: \mathbb{C} \otimes \mathbb{C} \rightarrow M_{2}(\mathbb{C})$ contains rank-one idempotents. It follows that $[e]-\left[e^{\prime}\right]=0$ in $K_{0}(\mathbb{C} \otimes R)$. To show that $[e]-\left[e^{\prime}\right]$ is an element of order two in $K_{0}(R)$ that is mapped to zero in $K_{0}(\mathbb{C} \otimes R)$ it suffices to show that the following theorem holds.

Theorem 10.1. The elements $[e]$ and $\left[e^{\prime}\right]$ are not equal in $K_{0}(R)$.

Proof. Let $T$ be the ring of real polynomial functions on the circle $x^{2}+y^{2}=1$. Parametrizing the circle by $t \in \mathbb{R} / \mathbb{Z}, T$ is generated as an $\mathbb{R}$-algebra by $c$ and $s$ defined as

$$
c(t)=\cos (2 \pi t), \quad s(t)=\sin (2 \pi t) .
$$

The ring $M_{2}(T)$ may be viewed as a ring of polynomial functions from the circle to $M_{2}(\mathbb{R})$. Define homomorphisms

$$
f_{i}: S_{i} \rightarrow M_{2}(T)
$$

by

$$
f_{1}(M)=(t \mapsto M), \quad f_{2}(M)=\left(t \mapsto f\left(\mathrm{e}^{\pi \mathrm{i} t}\right) M f\left(\mathrm{e}^{-\pi \mathrm{i} t}\right)\right),
$$

for any $M \in S_{i}$, where $f: \mathbb{C} \rightarrow M_{2}(\mathbb{R})$ is as above. Since the image of $f$ is commutative, $f_{1}$ and $f_{2}$ agree on the images of $\mathbb{C}$ in $S_{1}$ and $S_{2}$, and hence define a homomorphism $\hat{f}: R \rightarrow M_{2}(T)$. In fact, $\hat{f}$ is an isomorphism from $R$ to $M_{2}(T)$.

The homomorphisms $f_{1}$ and $f_{2}$ satisfy

$$
\begin{aligned}
f_{1}:\left(\begin{array}{ll}
1 & 0 \\
0 & 0
\end{array}\right) & \mapsto\left(\begin{array}{cc}
1 & 0 \\
0 & 0
\end{array}\right), \\
f_{2}:\left(\begin{array}{ll}
1 & 0 \\
0 & 0
\end{array}\right) & \mapsto\left(\begin{array}{cc}
(1+c) / 2 & s / 2 \\
s / 2 & (1-c) / 2
\end{array}\right) .
\end{aligned}
$$


Hence the images of $[e]$ and $\left[e^{\prime}\right]$ in $K_{0}(T)$ (under the composite of $f$ and the Morita equivalence map) are the classes represented by the free module of rank one and the ideal $(c+1, s)$, respectively. Since $(c+1, s)$ is a non-principal maximal ideal in the Dedekind domain $T$, it is not stably isomorphic to the free module (see 1.4.23 of [30]).

This argument is similar to that used in [19], except that there a topological argument (via the ring of continuous functions on the circle) was used to detect $[e]-\left[e^{\prime}\right]$.

Let $G$ be the double of the dihedral group $D_{p}$ of order $2 p$ along its subgroup $C_{p}$, for $p$ an odd prime. Recall that in Proposition 6.2 it was shown that

$$
K_{0}(\mathbb{R} G) \cong \mathbb{Z}^{\oplus(p+5) / 2} \oplus \mathbb{Z}_{2}^{\oplus(p-1) / 2} .
$$

The rank of $K_{0}(\mathbb{R} G)$ is equal to the number of conjugacy classes of elements of finite order in $G$, and so Hattori-Stallings traces detect $K_{0}(\mathbb{R} G)$ modulo its torsion subgroup. The torsion subgroup of $K_{0}(\mathbb{R} G)$ can be detected by homomorphisms from $\mathbb{R} G$ to the ring $R$ of Theorem 10.1, as follows.

Let $\rho$ be an irreducible two-dimensional real representation of the dihedral group $D_{p}$. Thus $\rho$ gives rise to a homomorphism $\rho: \mathbb{R} D_{p} \rightarrow M_{2}(\mathbb{R})$. It may be assumed that $\rho$ is orthogonal, in which case $\rho\left(C_{p}\right)$ is contained in $f(\mathbb{C}) \subseteq M_{2}(\mathbb{R})$. The corresponding $\mathbb{R}$-algebra homomorphisms

$$
\rho_{1}: \mathbb{R} D_{p} \rightarrow S_{1} \subseteq R, \quad \rho_{2}: \mathbb{R} D_{p} \rightarrow S_{2} \subseteq R
$$

coincide on $\mathbb{R} C_{p}$, and hence induce a homomorphism

$$
\hat{\rho}: \mathbb{R} G \rightarrow R
$$

There are $(p-1) / 2$ different choices of $\rho$. As an $\mathbb{R}$-algebra, $\mathbb{R} D_{p}$ is isomorphic to $\mathbb{R} \times \mathbb{R} \times M_{2}(\mathbb{R})^{\oplus(p-1) / 2}$, and the different choices of $\rho$ correspond to projections on to the $M_{2}(\mathbb{R})$ factors. This gives rise to $(p-1) / 2$ distinct homomorphisms from $\mathbb{R} G$ to $R$, and hence $(p-1) / 2$ homomorphisms from $K_{0}(\mathbb{R} G)$ to $K_{0}(R)$.

There is a $\mathbb{Z}_{2}$-basis for the torsion subgroup of $K_{0}(\mathbb{R} G)$ consisting of the elements $\left[e_{i}\right]-\left[e_{i}^{\prime}\right], 1 \leqslant i \leqslant(p-1) / 2$, where $e_{i}$ is a primitive idempotent in $\mathbb{R} D_{p}$ lying in the $i$ th copy of $M_{2}(\mathbb{R})$, and $e_{i}, e_{i}^{\prime}$ denote corresponding elements in the two copies of $\mathbb{R} D_{p}$ inside $\mathbb{R} G$. Each of these elements is sent to the element of order two in $K_{0}(R)$ by exactly one of the $(p-1) / 2$ homomorphisms from $\mathbb{R} G$ to $R$, and is sent to zero by the others. Hence the whole torsion subgroup of $K_{0}(\mathbb{R} G)$ is detected by these homomorphisms.

In the cases when either $k$ is a subfield of $\mathbb{R}$, or $k$ contains a primitive $p$ th root of unity, this gives an alternative method to detect all of the elements of $K_{0}(k G)$ given in Proposition 6.2. This suffices to prove cases (a) and (b) of Theorem 2.3.

\section{Stably free modules}

In this section, we exhibit non-free stably free finitely generated $k G$-modules for a certain virtually free group $G$ and any field $k$ of characteristic zero. Note that Bass has shown that when $G$ is free, every projective $k G$-module is free [1]. (This can also be deduced 
from the theorem of Bergman-Dicks quoted below as Theorem 11.1.) See $[\mathbf{3}, \mathbf{1 4}, \mathbf{2 0}]$ for examples of non-free stably free modules for group algebras of certain torsion-free groups.

For any ring $R$, let $\mathcal{P}(R)$ denote the abelian monoid of isomorphism types of finitely generated projective $R$-modules. A projective $R$-module $P$ is stably free of rank $r$ but not free if and only if $[P]=r[R]$ in $K_{0}(R)$, but $[P] \neq r[R]$ in $\mathcal{P}(R)$. As remarked earlier, the Bergman-Dicks approach to Theorem 4.1, which has the drawback that it requires stronger hypotheses than Waldhausen's, does have the advantage that it also gives the following.

Theorem 11.1. Let $G$ be the fundamental group of a finite graph of finite groups, and let $\mathcal{C}$ be a category with objects the edge and vertex groups in the graph for $G$, with (non-identity) morphisms the inclusions of each edge group in its initial and terminal vertex groups. Let $k$ be a field in which the order of each of the finite groups in $\mathcal{C}$ is a unit. Then the following natural map is an isomorphism:

$$
\operatorname{colim}_{H \in \mathcal{C}} \mathcal{P}(k H) \rightarrow \mathcal{P}(k G) .
$$

The corresponding statement for graphs of groups with larger edge groups is not valid. Lewin exhibits non-free stably free $\mathbb{Q} G$-modules for a class of groups which includes the fundamental groups of the trefoil knot and of the Klein bottle [20]. Dunwoody's non-free stably free $\mathbb{Z} G$-modules for $G$ the trefoil group [14] also give rise to non-free stably free $\mathbb{Q} G$-modules for this $G$. On the other hand, the trefoil and Klein bottle groups may be expressed as fundamental groups of graphs of groups with infinite cyclic edge and vertex groups, and every projective module for the rational group algebra of an infinite cyclic group is free.

Corollary 11.2. Let $G$ be the double of the alternating group $J=A_{5}$ along subgroups $H_{1}$ and $H_{2}$ of order two and three. For any field $k$ of characteristic zero there is a finitely generated projective $k G$-module $P$ such that $P \oplus k G$ is free, but for any $n>0, P^{\oplus n}$ is not free.

Proof. For any finite group $L$, the monoid $\mathcal{P}(k L)$ is isomorphic to a direct sum of copies of $\mathbb{N}$ indexed by the simple $k L$-modules, and embeds in $K_{0}(k L)$. (Of course, these assertions rely on the hypothesis that $k$ has characteristic zero.) For each choice of $k$, we shall exhibit finitely generated (projective) $k J$-modules $M_{1}$ and $M_{2}$ with the following properties.

(1) For some $r>0,\left[M_{1}\right]+\left[M_{2}\right]=r[k J]$ in $\mathcal{P}(k J)$.

(2) For each $n>0$ and each $1 \leqslant i, j \leqslant 2$, if $N \leqslant\left(M_{i}\right)^{\oplus n}$ and $N$ is isomorphic to a module induced up from $H_{j}$, then $N=0$.

(3) In $K_{0}$, for each $1 \leqslant i \leqslant 2,\left[M_{i}\right]$ is in the image of the induction map $K_{0}\left(k H_{1}\right) \oplus$ $K_{0}\left(k H_{2}\right) \rightarrow K_{0}(k J)$.

Given such $M_{i}$, let $J_{1}$ and $J_{2}$ be the two copies of $J$ used to build $G$, and define $P=\operatorname{Ind}_{J_{1}}^{G}\left(M_{1}\right) \oplus \operatorname{Ind}_{J_{2}}^{G}\left(M_{2}\right)$. Then by (1) and (3), it follows that in $K_{0}(k G)$,

$$
[P]=\operatorname{Ind}_{J_{1}}^{G}\left(\left[M_{1}\right]+\left[M_{2}\right]\right)=\operatorname{Ind}_{J_{1}}^{G}\left(r\left[k J_{1}\right]\right)=r[k G] .
$$


On the other hand, the description of $\mathcal{P}(k G)$ given by Theorem 11.1 and property (2) imply that for each $n>0, n[P] \neq n r[k G]$ in $\mathcal{P}(k G)$.

It remains to exhibit $M_{1}, M_{2}$ satisfying (1)-(3). Firstly, suppose that $k$ is a splitting field for $J$ and for the $H_{j}$. (This occurs if and only if $k$ contains $\omega$, a primitive cube root of 1 , and $\sqrt{5}$.) In this case, there are five simple $k J$-modules, which we shall denote by $\chi_{1}, \ldots, \chi_{5}$, of $k$-dimensions $1,3,3,4$ and 5 , respectively. The modules

$$
M_{1}=\chi_{1}+\chi_{4}+5 \chi_{5}, \quad M_{2}=3 \chi_{2}+3 \chi_{3}+3 \chi_{4}
$$

have properties (1)-(3), and give rise to a non-free stably free $k G$-module of rank one. The same elements will suffice if $k$ contains $\omega$ but does not contain $\sqrt{5}$, except that in this case there are only four simple $k J$-modules, and $\chi_{2}+\chi_{3}$ should be understood to denote a simple $k J$-module of dimension six. When $k$ does not contain $\omega$, the $M_{i}$ as above do not satisfy (3). However, the modules $M_{i}^{\prime}=M_{i} \oplus M_{i}$ satisfy (1)-(3) and may be used to construct a $k G$-module that is not free and is stably free of rank two.

Of course the method used in proving Corollary 11.2 could be applied to the fundamental groups of many other graphs of finite groups, but we preferred to concentrate on a specific example.

Acknowledgements. The author acknowledges support from the Nuffield Foundation, the CRM Barcelona, and from EPSRC grants GR/L06928, GR/L69398 and GR/R07813. This work grew out of a study of the contents of [18]. Some examples with similar properties to ours were constructed using other methods in Boaz Moselle's thesis [26]. The author thanks Alejandro Adem, Noel Brady, Ken A. Brown, Warren Dicks and Peter Kropholler for various helpful remarks concerning this work. Finally, the author thanks the anonymous referee, who found more than one error in an earlier version of this paper and also made various suggestions for improving its exposition.

\section{References}

1. H. BASs, Projective modules over free groups are free, J. Algebra 1 (1964), 367-373.

2. G. M. Bergman, Modules over coproducts of rings, Trans. Am. Math. Soc. 200 (1974), $1-32$.

3. P. H. Berridge and M. J. Dunwoody, Nonfree projective modules for torsion-free groups, J. Lond. Math. Soc. 19 (1979), 433-436.

4. M. Bestvina, Local homology properties of boundaries of groups, Michigan Math. J. 43 (1996), 123-139.

5. M. Bestvina And N. Brady, Morse theory and finiteness properties of groups, Invent. Math. 129 (1997), 445-470.

6. R. Bieri, Gruppen mit Poincaré Dualität, Comment. Math. Helv. 47 (1972), 373-396.

7. R. BIERI, Homological dimension of discrete groups, Queen Mary College Mathematics Notes (University of London, 1976).

8. K. S. Brown, Cohomology of groups, Graduate Texts in Mathematics, vol. 87 (Springer, 1982).

9. K. A. BRown AND M. Lorenz, Colimits of functors, and Grothendieck groups of infinite group algebras, Contemp. Math. 130 (1992), 98-109.

10. C. W. Curtis and I. Reiner, Methods of representation theory, vol. 1 (Wiley, 1981). 
11. M. W. DAvis, Poincaré duality groups, in Surveys on surgery theory, vol. 1, in Annals of Mathematics Studies, vol. 145 (ed. S. Cappell, A. Ranicki and J. Rosenberg) (Princeton University Press, Princeton, NJ, 2000).

12. W. Dicks, The HNN construction for rings, J. Algebra 81 (1983), 434-487.

13. W. Dicks And M. J. Dunwoody, Groups acting on graphs, Cambridge Studies in Advanced Mathematics, vol. 17 (Cambridge University Press, 1989).

14. M. J. Dunwoody, Relation modules, Bull. Lond. Math. Soc. 4 (1972), 151-155.

15. S. Gersten, A product formula for Wall's obstruction, Am. J. Math. 88 (1966), 337-346.

16. J. E. HumphreYs, Reflection groups and Coxeter groups, Cambridge Studies in Advanced Mathematics, vol. 29 (Cambridge University Press, 1990).

17. F. E. A. Johnson And C. T. C. Wall, On groups satisfying Poincaré duality, Ann. Math. 96 (1972), 592-598.

18. P. H. Kropholler And B. Moselle, A family of crystallographic groups with 2-torsion in $K_{0}$ of the rational group algebra, Proc. Edinb. Math. Soc. 34 (1991), 325-331.

19. I. J. LeARY, A torsion projective class for a group algebra, Bull. Lond. Math. Soc. 32 (2000), 75-77.

20. J. LEWIN, Projective modules over group algebras of torsion-free groups, Michigan Math. J. 29 (1982), 59-64.

21. M. Lorenz, The rank of $G_{0}$ for polycyclic group algebras, in Topics in algebra, part 1, pp. 45-53 (Banach Center Publications, PWN, Warsaw, 1990).

22. M. Lorenz, Picard groups of multiplicative invariants, Comment. Math. Helv. 72 (1997), 389-399.

23. W. LÜCK, Chern characters for proper equivariant homology theories and applications to K- and L-theory, J. Reine Angew. Math. 543 (2002), 193-234.

24. G. MisLin, Wall's finiteness obstruction, in Handbook of algebraic topology, pp. 1259-1291 (North-Holland, Amsterdam, 1995).

25. J. A. Moody, Induction theorems for infinite groups, Bull. Am. Math. Soc. 17 (1987), 113-116.

26. B. Moselle, Homological properties of polycyclic group algebras, PhD thesis, Queen Mary College, London (1990).

27. J. R. Munkres, Elements of algebraic topology (Addison-Wesley, 1984).

28. D. S. PASSMAn, The algebraic structure of group rings (Wiley Interscience, 1977).

29. F. Quinn, Algebraic K-theory of poly (finite or cyclic) groups, Bull. Am. Math. Soc. 12 (1985), 221-226.

30. J. Rosenberg, Algebraic K-theory and its applications, Graduate Texts in Mathematics, vol. 147 (Springer, 1994).

31. F. WALDHAUSEN, Whitehead groups of generalised free products, Algebraic K-theory, vol. II, pp. 155-179, Lecture Notes in Mathematics, vol. 342 (Springer, 1973).

32. F. Waldhausen, Algebraic $K$-theory of generalized free products, parts I and II, Ann. Math. 108 (1978), 135-204, 205-256.

33. C. T. C. WALl, Finiteness conditions for CW-complexes, Ann. Math. 81 (1965), 56-69.

34. C. T. C. WAll, Finiteness conditions for CW-complexes, II, Proc. R. Soc. Lond. A 295 (1966), 129-139.

35. C. T. C. Wall, Poincaré complexes, I, Ann. Math. 86 (1967), 213-245. 